\title{
Mechanical Embolectomy for Acute Ischemic Stroke in the Anterior Cerebral Circulation: The Gothenburg Experience during 2000-2011
}

\author{
A. Rentzos, C. Lundqvist, J.-E. Karlsson, V. Vilmarsson, K. Schnabel, and G. Wikholm
}

\begin{abstract}
BACKGROUND AND PURPOSE: Intra-arterial treatment of proximal occlusions in the cerebral circulation have become an important tool in the management of acute ischemic stroke. Our goal was to evaluate the safety and efficacy of intra-arterial acute ischemic stroke treatment performed in our institution in consecutive patients with anterior circulation occlusion during 2000-2011.
\end{abstract}

MATERIALS AND METHODS: We identified, in our data base, 156 consecutive cases with anterior acute ischemic stroke treated intraarterially during 2000-2011. Stroke severity was defined according to the National Institutes of Health Stroke Scale, the results of the procedure were defined according to the modified Thrombolysis in Cerebral Infarction score, and clinical outcome was defined according to the modified Rankin scale, with favorable outcome $\leq 2$ at 90 days.

RESULTS: The mean admission NIHSS score was 19.4 (median, 20), with a mean time from stroke onset to groin puncture of 197 minutes (median, 171 minutes). The embolectomy tool of choice was the Amplatz GooseNeck snare (83\%). Successful recanalization (modified TICI $2 b+3$ ) was seen in $74 \%$ of cases. A mRS $\leq 2$ at 90 days was seen in $42 \%$ with a mortality rate of $17 \%$ and symptomatic intracerebral hemorrhage in $4 \%$.

CONCLUSIONS: A high recanalization rate was obtained with the Amplatz GooseNeck snare without any device-related complications. Favorable outcome, mortality, and symptomatic intracerebral hemorrhage are comparable with results of newer embolectomy devices.

ABBREVIATIONS: AIS = acute ischemic stroke; $\mathrm{mTICI}=$ modified Thrombolysis in Cerebral Infarction; sICH = symptomatic intracerebral hemorrhage

$\mathrm{T}$

reatment with intravenous rtPA has proved successful in acute ischemic stroke within 4.5 hours of stroke onset. ${ }^{1}$ However, in patients presenting with a high NIHSS score and proximal occlusions, intravenous treatment has been less successful, with low recanalization rates and increased mortality. ${ }^{2-5}$ In the quest for more effective recanalization of proximal occlusions, research in intra-arterial treatment of acute ischemic stroke (AIS) has been ongoing for decades. It started with the intra-arterial injection of rtPA but made an important breakthrough in more recent years with the advent of stent-based embolectomy, especially with the Solitaire FR device (Covidien, Irvine, California), which had a huge impact in the intra-arterial treatment of AIS by substantially improving the recanalization rate. The success of this stent-

Received November 17, 2013; accepted after revision March 11, 2014.

From the Departments of Interventional and Diagnostic Neuroradiology (A.R., V.V., K.S., G.W.) and Neurology (C.L., J.-E.K.), Sahlgrenska University Hospital, Gothenburg, Sweden.

Please address correspondence to Alexandros Rentzos, MD, Interventional Neuroradiology, Sahlgrenska University Hospital, 41345 Gothenburg, Sweden; e-mail: alexandros.rentzos@vgregion.se

http://dx.doi.org/10.3174/ajnr.A3997 based embolectomy approach continued with the production of other embolectomy devices such as Trevo (Stryker, Kalamazoo, Michigan) or Revive SE (Codman Neurovascular, Raynham, Massachusetts), which differ in characteristics but are based on the same principle as the Solitaire FR. At the same time, this breakthrough, along with increased competitiveness in the medical industry, brought an improvement of older embolectomy devices such as the Merci retriever (Concentric Medical, Mountain View, California) and the Penumbra System (Penumbra, Alameda, California). Our institution has been actively involved in intra-arterial treatment of AIS, performing intra-arterial thrombolysis and mechanical embolectomies since the beginning of the 1990s.

The Amplatz GooseNeck snare (Covidien) is a very common tool used in interventional radiology for extraction of foreign objects via the endovascular route, but it was first presented as an embolectomy tool in $1998 .^{6}$ Extracting a coherent but still softer material such as an embolus with the Amplatz GooseNeck snare involves slight moderation in the technique used for extracting foreign objects. ${ }^{7}$ The aim of this retrospective study was to assess the safety and efficacy of these intra-arterial treatment methods in 
Table 1: Group characteristics

\begin{tabular}{|c|c|c|}
\hline Age (yr), mean (median) & \multicolumn{2}{|c|}{$64 \pm 13.9(67)$} \\
\hline Sex & \multicolumn{2}{|c|}{$63 \%$ men, $37 \%$ women } \\
\hline Side of occlusion & \multicolumn{2}{|c|}{$60 \%$ left, $40 \%$ right } \\
\hline \multicolumn{3}{|l|}{ Site of occlusion } \\
\hline Distal ICA & 2 & \multirow{3}{*}{$10(6 \%)$} \\
\hline Distal ICA+MI & 6 & \\
\hline Distal ICA+M2 & 2 & \\
\hline Car-T & & $45(29 \%)$ \\
\hline M1 & 77 & \multirow{2}{*}{$84(54 \%)$} \\
\hline $\mathrm{M} 1+\mathrm{M} 2$ and/or $\mathrm{A} 2 / \mathrm{A} 3$ & 7 & \\
\hline $\mathrm{M} 2$ & 13 & \multirow[t]{2}{*}{$17(11 \%)$} \\
\hline $\mathrm{M} 2$ + other distal emboli & 4 & \\
\hline \multicolumn{3}{|l|}{ Cause of stroke } \\
\hline Carotid stenosis & \multicolumn{2}{|c|}{17 (11\%) } \\
\hline Dissection & \multicolumn{2}{|c|}{$12(8 \%)$} \\
\hline Cardiac & \multicolumn{2}{|c|}{$95(61 \%)$} \\
\hline No identifiable cause & \multicolumn{2}{|c|}{29 (19\%) } \\
\hline Periprocedural stroke & \multicolumn{2}{|c|}{$3(2 \%)$} \\
\hline NIHSS score, mean (median) & \multicolumn{2}{|c|}{$19.4 \pm 4.2(20)$} \\
\hline
\end{tabular}

Note:-Car-T indicates carotid- $T$.

patients with severe anterior AIS. The patients were treated in our department during 2000-2011; most of the treatments were performed with the Amplatz GooseNeck snare.

\section{MATERIALS AND METHODS}

Intra-arterial AIS treatment has been performed in our institution since the early 1990s, initially in the form of intra-arterial thrombolysis and later mechanically, with the addition of embolectomy with the Amplatz GooseNeck snare. The criteria for intra-arterial AIS therapy included no intracerebral hemorrhage on the admission NCCT, NIHSS score of $\geq 14$ when no CTA was performed, proved occlusion on CTA when performed, and treatment initiation within 6 hours. Hypoattenuation in more than one-third of the MCA would not exclude a patient from intra-arterial treatment except in cases in which hypoattenuation extended into most of the MCA territory. Patients admitted to our hospital who were eligible for intra-arterial treatment did not receive intravenous thrombolysis but were transported directly to the neurointerventional suite. In 2009, an intravenous bolus dose of rtPA before intra-arterial treatment was added to the protocol. Bridging therapy with a bolus dose and a continuous infusion of intravenous $\mathrm{rtPA}(0.9 \mathrm{mg} / \mathrm{kg}$; maximum, $90 \mathrm{mg}$ ) was reserved for patients referred to us from other regional hospitals. As a rule, intra-arterial AIS treatment was performed with the patient under general anesthesia. For embolectomy, we mainly used the Amplatz GooseNeck snare but also the Solitaire FR revascularization device, the Merci retriever, and the Catch thrombectomy device (Balt, Montmorency, France). Intra-arterial injection of rtPA locally at the site of occlusion was mainly reserved as a complement to embolectomy or for more distally located emboli. Intra-arterial thrombolysis was rarely used as a primary method, and it was mainly performed earlier in the study period. Overall a combination of different tools was often used.

In our prospective data base, in which all patients treated in our unit are registered, 179 patients who had undergone intraarterial treatment for anterior AIS since 1991 were identified. One hundred fifty-six such procedures (in 155 patients) met the following inclusion criteria: All patients presented with AIS with an
Table 2: Time intervals

\begin{tabular}{lc}
\hline & Mean \pm SD (Median) \\
\hline Stroke to groin puncture & $197 \pm 81 \mathrm{~min}$ (171 min) \\
Stroke to recanalization & $289 \pm 87 \mathrm{~min}(288 \mathrm{~min})$ \\
Stroke to CT & $100 \pm 54 \mathrm{~min}(83 \mathrm{~min})$ \\
CT to groin puncture & $98 \pm 65 \mathrm{~min}(79 \mathrm{~min})$ \\
Procedure (1st-last image) & $102 \pm 87 \mathrm{~min}(92 \mathrm{~min})$ \\
\hline
\end{tabular}

Table 3: Recanalization grade and functional outcome

\begin{tabular}{lr}
\multicolumn{1}{c}{$\mathbf{m T I C I}$} & \multicolumn{1}{c}{ mRS } \\
\hline $0=27(17 \%)$ & $0-1=37(24 \%)$ \\
$1=5(3 \%)$ & $\leq 2=65(42 \%)$ \\
$2 a=9(6 \%)$ & $3=29(19 \%)$ \\
$2 b=81(52 \%)$ & $4-5=35(22 \%)$ \\
$3=34(22 \%)$ & $6=27(17 \%)$ \\
\hline
\end{tabular}

occlusion in a major artery as described in Tables $1-3$. The time of symptom onset was known for all patients, and they had an NIHSS score of $\geq 14$ when no CTA was performed or a proved occlusion on CTA. All pre- and postprocedural radiologic data were available and could be retrieved from the hospital archive. The functional status according to mRS was obtained by a vascular neurologist. In cases in which the mRS was obtained by a nonvascular neurologist or the score was not registered in the medical charts, 1 of our 2 experienced vascular neurologists would contact the patient or close relatives to confirm or obtain the mRS. The radiologic images were reviewed by 2 neurointerventionalists and trained neuroradiologists, and the entire process was under the supervision of a senior neurointerventionalist. In case of disagreement between the 2 reviewers, a repeat review with the supervisor was performed and a consensus-based solution was established. The vascular neurologists were blinded to the modified Thrombolysis in Cerebral Infarction (mTICI) score and the neurointerventionalists, to the mRS.

In the study protocol, we documented the NIHSS score at admission, use of intravenous rtPA, use of general anesthesia, extent of hypoattenuation in NCCT, location of the occlusion in CTA and DSA, use of embolectomy device, recanalization score according to the mTICI scale, complications, functional status according to the mRS at 3 months, all time intervals from stroke onset and NCCT examination to groin puncture, recanalization, and the last image of the procedure. In case of death in the 3-month period, the cause of mortality was established.

Statistical evaluations were performed with the Statistical Package for the Social Sciences, Version 22 (IBM, Armonk, New York) by using descriptive statistics (means, percentages, SDs) for the variables. The $\chi^{2}$ or the Fisher exact test with a $P$ level $<.05$ indicating a statistically significant difference was used for comparisons as appropriate.

Institutional review board approval was obtained to review the radiologic and clinical data.

\section{RESULTS}

\section{Preprocedural Diagnostic Work-Up}

From the 155 patients who fulfilled the study inclusion criteria, 10 had an NIHSS score of $<14$ but a proved occlusion in the CTA. NCCT of the head was the initial diagnostic method in all cases except 3 . Among the 3 patients with no NCCT, 1 had MR imaging 


\begin{tabular}{lccc}
\hline & mTICl & mRS $<2$ & mRS 6 \\
\hline Hyperattenuated MCA sign 114/153 (74.5\%) & $2 \mathrm{~b}+3: 86(75 \%)$ & $44(39 \%)$ & $23(20 \%)$ \\
Hypoattenuation less than one-third of MCA, 119 (78\%) & $0-2 \mathrm{a}: 31(26 \%)$ & $6(19 \%)$ & $10(32 \%)$ \\
& $2 \mathrm{~b}+3: 88(74 \%)$ & $49(56 \%)$ & $5(6 \%)$ \\
Hypoattenuation more than one-third of MCA, 34 (22\%) & $0-2 \mathrm{a}: 15(44 \%)$ & $1(15 \%)$ & $6(40 \%)$ \\
& $2 \mathrm{~b}+3: 19(56 \%)$ & $8(42 \%)$ & $6(32 \%)$ \\
\hline
\end{tabular}

and 2 had complications of carotid surgery and percutaneous coronary intervention, respectively.

Table 4 shows the findings of the preprocedural NCCT. Most (78\%) had hypoattenuation in less than one-third of the MCA territory. If successful recanalization was achieved, a favorable outcome was seen in $56 \%$ of patients with hypoattenuation in less than one-third of the MCA territory and, remarkably, in $42 \%$ of patients with more than one-third hypoattenuation $(P=.67)$. However, the mortality rate was $6 \%$ with less than one-third hypoattenuation and $32 \%$ with more than one-third hypoattenuation, respectively $(P=.02)$. The cause of mortality in the second group, though, was mainly due to unrelated diseases and, in only 2 subjects, due to malignant infarction. The unrelated diseases were one of the following; uncontrolled pulmonary hypertension in a patient with cardiomyopathy, aspiration pneumonia, new stroke in the contralateral MCA in the following days, and perforation during the procedure.

When treatment was unsuccessful, the mortality rate was $32 \%$ in patients with hypoattenuation in less than one-third of the MCA territory, and it reached $40 \%$ in patients with hypoattenuation of more than one-third, most in this case attributed to malignant infarction and/or symptomatic intracerebral hemorrhage (sICH).

In 92 of the 153 cases, mainly those treated during the second half of the period (2006-2011), the diagnostic imaging protocol included a CTA besides the initial NCCT and the site of intracranial occlusion could be identified in all cases with CTA.

\section{Intra-Arterial Treatment}

Most patients were treated under general anesthesia except 14 cases treated under conscious sedation. In 2 cases, the treatment began with the patient under conscious sedation but was converted to general anesthesia. The treatment was initiated within 6 hours from the onset of symptoms in all cases except in 1 in which the treatment started 7 hours after the onset.

The Amplatz GooseNeck snare was used exclusively in 129 cases (83\%) as the first device and as second or third device in 6 cases. In 25 cases, it was used to retrieve more distally located significant emboli. Successful recanalization was achieved with the Amplatz GooseNeck snare in $70 \%$ of the cases where it was the primary tool (90/129) while in all cases of successful recanalization $(n=115)$ the Amplatz GooseNeck snare was the embolectomy tool in $78 \%(90 / 115)$. No device-related complications could be identified with this snare. During 2000-2005, it was not always used as a first method by all neurointerventionalists because intra-arterial thrombolysis quite often was the first choice. The recanalization rate during this period was only $52 \%$. When the Amplatz GooseNeck snare technique was established as the first method among all neurointerventionalists, the recanalization rate increased to $82 \%$ (during 2006-2011).
The Solitaire FR was used in 33 cases, with a recanalization rate of $42 \%$ (14/33), while the third most common device was the Merci retriever, used in 18 cases with a recanalization rate of $30 \%$ (6/18). However, these devices were mainly used after unsuccessful recanalization with the Amplatz GooseNeck snare and, only in a few cases, as first devices, usually during testing periods when these devices were new.

Intra-arterial thrombolysis with $\mathrm{rtPA}$ as the sole treatment method was used in 13 patients with a recanalization rate of $46 \%$ (6/13).

A combination of different devices was used in 31\% (49/156). In 22 of the 49 cases, the occlusion was in the distal internal carotid or it was a carotid-T occlusion (occlusion in the distal internal carotid artery involving the $\mathrm{A} 1$ and $\mathrm{M} 1$ segments), while in 12 cases, a combination of devices was needed to extract residual emboli in the M2 and M3 segments or new emboli most often in the $\mathrm{A} 1$ and $\mathrm{A} 2$ segments.

\section{Complications}

Serious complications were seen in 8 cases (5\%), with 4 being carotid-T occlusions and 4, M1 segment occlusions. The complications were perforations in 5 cases and dissections in 3 cases. Four patients died due to complications. Those who survived had $\operatorname{mRS} 2(n=1), 3(n=2)$, and $4(n=1)$.

In 4 of the 5 perforation cases, the cause was the microguidewire, and in 2, perforation occurred while trying to reach the occluded vessel via the contralateral circulation through the anterior communicating artery. One of these cases showed no visible subarachnoid hemorrhage in the postprocedural NCCT, despite a documented mild extravasation of contrast on DSA. In the fifth perforation case, a significant stenosis with poor filling of the vascular bed distal to the occlusion was detected in the M1 segment after a successful embolectomy. Attempted balloon dilation in this case resulted in a perforation of the MCA.

Two of the 3 dissections happened during embolectomy with the Merci retriever with subsequent bleeding seen in the postprocedural NCCT in 1 case. In the third case, the dissection was seen after a pass with the Solitaire FR through an M1 occlusion. A control DSA in this case revealed a possible stenosis and probable SAH, but no postprocedural NCCT was available for this patient.

Treatment of severe vasospasm in the M1 segment with local nimodipine infusion was used in 3 cases, with regression of the vasospasm in 2 cases, while in the third, the vasospasm after embolectomy with the Merci retriever was substantial and was so severe that only minimal flow could be seen distal to the vasospasm with no filling of the vascular bed.

\section{Postprocedural Radiologic and Neurologic Results}

In 150 cases, a postprocedural NCCT was available, with a mean acquisition time of 26 hours 31 minutes from the end of the DSA. 
An mRS score was available in all 155 patients, with $\mathrm{mRS} \leq 2$ in $42 \%(65 / 155)$. In 115 cases with $\mathrm{mTICI} 2 \mathrm{~b} / 3, \mathrm{mRS} \leq 2$ was seen in $51 \%(59 / 115)$. One patient had 2 stroke episodes and received intra-arterial therapy twice. Bad functional outcome (mRS 3-6) was as high as $85 \%$ in patients with unsuccessful recanalization, decreasing dramatically to $49 \%$ in patients with successful recanalization $(P<.01)$. The mortality rate (mRS 6) after 3 months was $17 \%$. Death related directly to the ischemic stroke and the endovascular procedure was observed in 17 patients (11\%), while death from other causes was found in 10 patients (6\%). Comparing the mortality rate in patients with unsuccessful and successful recanalization yielded a significant difference of $39 \%(16 / 41)$ and $10 \%(11 / 115)$, respectively $(P<.01)$.

Dividing the patients into a group with a stroke onset-togroin puncture time of $<180$ minutes and a group with a time of $>180$ minutes resulted in $80 \%$ recanalization rate and a $42.5 \%$ $\mathrm{mRS} \leq 2$ for the first group and a $67 \%$ recanalization rate $(P=$ $.07)$ and a $41 \% \mathrm{mRS} \leq 2(P=.8)$ for the second group. The same analysis, except using 270 minutes as a cutoff point, yielded the same recanalization rate of $74 \%$ for both groups $(P=.98)$ and an $\mathrm{mRS} \leq 2$ with only a slight difference, $43 \%$ for the group with stroke onset-to-groin puncture of $<270$ minutes and $41 \%$ for $>270$ minutes $(P=.47)$.

The rate of sICH, as defined in the Safe Implementation of Thrombolysis in Stroke-Monitoring Study criteria was difficult to calculate due to lack of an early postprocedural NIHSS estimation in some patients. The presence of $\mathrm{PH} 2$ and review of the medical charts for a description of obvious deterioration in the patient's status were used with sICH estimated at $4 \%$. The patients with sICH were equally distributed in the groups with stroke onset-togroin puncture time under and over 180 minutes and equally distributed in groups showing hypoattenuation in less and more than one-third of the MCA territory. In 5 patients, SAH was identified and attributed to procedural complications as described earlier, except in 1 case in which a traumatic SAH was identified during review of the preprocedural NCCT.

Sixteen patients identified in 2000-2011 received bridging therapy. Successful recanalization was seen in $75 \%$, while $\mathrm{mRS} \leq$ 2 was seen in $31 \%(P=.4)$. Bleeding was seen in only 3 patients who received bridging therapy, and none were symptomatic.

A subanalysis of patients with carotid-T occlusion (45/156) shows that carotid- $\mathrm{T}$ occlusions have the lowest recanalization rate $(55.5 \%)$ with a favorable outcome of $33 \%$ and a mortality rate of $22 \%$. Patients presenting with M1 and/or M2 occlusions showed a higher recanalization rate $(83 \%)$ compared with carotid-T occlusions $(P=.01)$, an $\mathrm{mRS} \leq 2$ at $46 \%$, and lower mortality rate $(13 \%)$ than the average mortality rate of all patients in the study $(17 \%)$.

\section{DISCUSSION}

Our study shows that a high recanalization rate can be achieved with the Amplatz GooseNeck snare, which can be used fast and safely for restoration of intracerebral circulation. The Amplatz GooseNeck snare technique was not established among neurointerventionalists in our department from the beginning, and commonly, intra-arterial thrombolysis was used as the first method. When the Amplatz GooseNeck snare became the standard embo- lectomy method and the stroke logistics were restructured in our hospital leading to an increased proportion of patients receiving intra-arterial treatment, the recanalization rate reached $82 \%$ (2006-2011). During this time, a number of new devices were tried in our department, most commonly the Merci retriever first and later the Solitaire FR, the latter being, for the moment, our second device. The Amplatz GooseNeck snare is kept as the device of choice because newer devices have not shown any better results, are more expensive, and have shown a higher complication rate. Moreover, the catheterization time was not longer than in newer devices and may be faster, given that no incubation time is required after deployment and before the retrieval of the device (Fig $1)$.

Lastly, no procedural complications could be identified with the Amplatz GooseNeck snare, while a number of complications occurred with other devices or with the guidewire while trying to bypass the occlusion, something that it is not required with the Amplatz GooseNeck snare. However, the Solitaire FR, Merci retriever, and the other devices in our study were used as the first choice in only a few cases, usually during the test period for each device. As a rule, we used these devices only when recanalization was unsuccessful with the Amplatz GooseNeck snare. In most cases, these devices did not succeed either, leading to the low recanalization scores presented in the "Results." Studies in which these devices are the first choice show high recanalization rates and are more representative of the stent retrievers' true efficacy. ${ }^{8}$

The Amplatz GooseNeck snare reaches recanalization rates comparable with the newer stent retriever and, at the same time, offers advantages like cost-effectiveness and low complication rates, which make it a feasible and reliable embolectomy device, increasing the device choices available in the treatment of AIS.

Our study represents consecutively treated patients who received intra-arterial AIS treatment based mainly on clinical criteria of eligibility. Hypoattenuation in more than one-third of the MCA territory on NCCT was not an absolute contraindication. Many patients who would otherwise have been excluded by using the one-third hypoattenuation rule or even the ASPECTS as an absolute criterion were classified as eligible for intra-arterial treatment in our institution. The analysis of the preprocedural diagnostic work-up shows that there is a group of patients with hypoattenuation in more than one-third of the MCA territory who do benefit from successful recanalization. This patient group has a high mortality rate, but the causes of mortality in this group were, in most, not related to malignant infarct or sICH. Failing, however, to restore the circulation in this patient group leads to a slightly higher mortality rate and, more important, the causes of mortality are mostly related to malignant infarct and/or sICH. Using one-third of the MCA territory as the cutoff point for patient selection would have improved the total mRS of the group but, at the same time, would exclude many eligible patients with a poor prognosis who might benefit from the treatment, either reaching a good functional outcome or at least surviving. This highlights the necessity of improving our selection criteria.

The worst recanalization rate was seen in patients with carotid-T occlusion. A combination of different devices was often used in this type of occlusion. Good functional outcome was reached only after a successful recanalization in all but 1 of the 

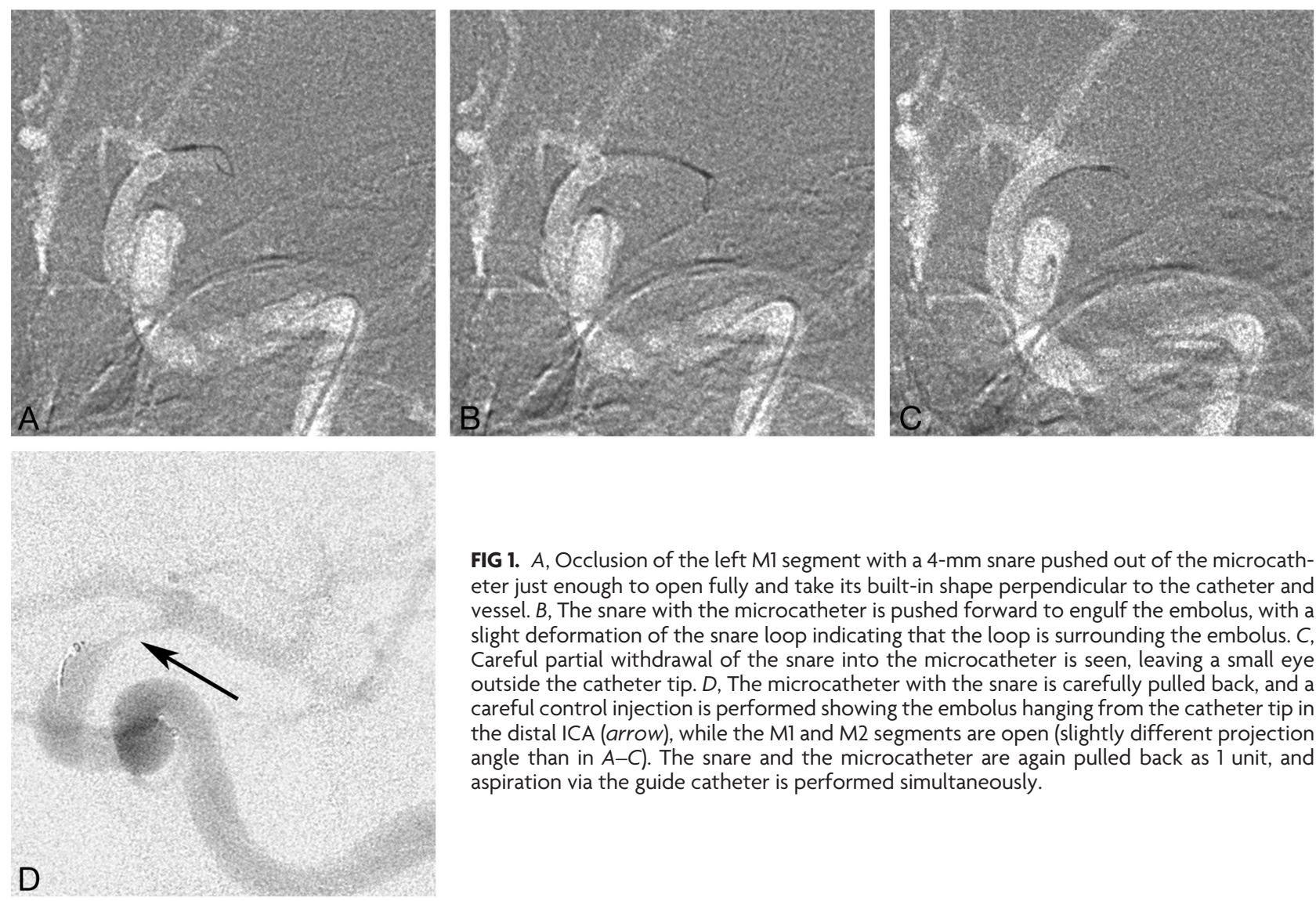

FIG 1. A, Occlusion of the left $\mathrm{M} 1$ segment with a 4-mm snare pushed out of the microcatheter just enough to open fully and take its built-in shape perpendicular to the catheter and vessel. $B$, The snare with the microcatheter is pushed forward to engulf the embolus, with a slight deformation of the snare loop indicating that the loop is surrounding the embolus. $C$, Careful partial withdrawal of the snare into the microcatheter is seen, leaving a small eye outside the catheter tip. $D$, The microcatheter with the snare is carefully pulled back, and a careful control injection is performed showing the embolus hanging from the catheter tip in the distal ICA (arrow), while the M1 and M2 segments are open (slightly different projection angle than in $A-C)$. The snare and the microcatheter are again pulled back as 1 unit, and aspiration via the guide catheter is performed simultaneously.

patients with carotid-T, making recanalization the only possibility for a good outcome in these patients.

Our study shows that successful recanalization is essential to reaching independence and significantly decreases the mortality rate, while the 180- or 270-minute cutoff point from stroke onset to groin puncture does not significantly influence the recanalization rate, the neurologic outcome, or the occurrence of sICH.

Treating patients with occlusion of a major proximal artery with intravenous rtPA alone can be successful in some cases but usually leads to unsuccessful or delayed recanalization, followed by increased morbidity and mortality rates. ${ }^{2-5}$ The efficacy of the mechanical embolectomy has never been validated through randomized trials until recently, when the results of the Interventional Management of Stroke III and Synthesis Expansion randomized studies were published. ${ }^{9,10}$ Both studies compared intraarterial treatment of AIS with intravenous thrombolysis alone but were not able to prove the superiority of the intra-arterial approach. However, in these studies, only intra-arterial thrombolysis and mostly older-generation devices were used, with a poor-to-moderate recanalization outcome. In addition, a preprocedural CTA to prove large-vessel occlusion was not performed in most patients; this procedure should be a prerequisite in such randomized trials. ${ }^{11,12}$ There is, thus, a need for further investigation of the efficacy of the intra-arterial stroke treatment with a newer generation of devices such as stent retrievers and devices with proven good recanalization rates. Furthermore, unbiased comparison of the 2 methods requires that group characteristics and stroke severity be equal for both arms of treatmentthat is, an NIHSS score between 10 and 24 points with proved occlusion, excluding patients with a low NIHSS score where intra-arterial treatment is not indicated, and patients with $>25$ NIHSS points when intravenous thrombolysis is contraindicated.

While a satisfactory recanalization rate of occlusion in major arteries can be achieved with the Amplatz GooseNeck snare and the newer embolectomy devices, ${ }^{13,14}$ further research is also needed to improve the selection of patients eligible for intra-arterial treatment. We recently added CT perfusion to our diagnostic protocol in an effort to better detect patients with an established infarct core and no or minimal penumbra to rescue and even patients with a large infarcted area who can eventually be at risk for $\mathrm{sICH}$ after a successful recanalization. ${ }^{15}$ With this approach, we also hope to identify patients outside the present time window or with an unknown time of onset (eg, wake-up stroke) who may benefit from intra-arterial treatment.

Continuous effort to minimize the time between stroke onset and treatment initiation along with a rising public awareness of stroke symptoms would further contribute to a decrease in the morbidity and mortality rates.

\section{CONCLUSIONS}

Embolectomy with the Amplatz GooseNeck snare is safe and effective, reaching recanalization rates and functional outcome comparable with results of the newer embolectomy devices. Patients presenting with hypoattenuation in more than one-third of the MCA territory might benefit from intra-arterial treatment and should not be excluded. Successful recanalization is the most essential parameter for a good clinical outcome and survival of 
these patients; improvement of the selection process of patients eligible for intra-arterial AIS treatment, good organization of the stroke unit, and rising public awareness will improve the clinical outcome in the future. However, there is a need of further validation of mechanical embolectomy, which will require new randomized studies.

Disclosures: Christer Lundqvist-RELATED: He consults for Agito, and part of his salary supported travel to the European Stroke Congress in Nice (May 2014).

\section{REFERENCES}

1. Wahlgren N, Ahmed N, Dávalos A, et al. Thrombolysis with alteplase 3-4.5 h after acute ischaemic stroke (SITS-ISTR): an observational study. Lancet 2008;372:1303-09

2. Bhatia $\mathrm{R}, \mathrm{Hill} \mathrm{MD}$, Shobha $\mathrm{N}$, et al. Low rates of acute recanalization with intravenous recombinant tissue plasminogen activator in ischemic stroke: real-world experience and a call for action. Stroke 2010;41:2254-58

3. Porelli S, Leonardi M, Stafa A, et al. CT angiography in an acute stroke protocol: correlation between occlusion site and outcome of intravenous thrombolysis. Interv Neuroradiol 2013;19:87-96

4. Smith WS, Lev MH, English JD, et al. Significance of large vessel intracranial occlusion causing acute ischemic stroke and TIA. Stroke 2009;40:3834-40

5. Rangaraju S, Owada K, Noorian AR, et al. Comparison of final infarct volumes in patients who received endovascular therapy or intravenous thrombolysis for acute intracranial large-vessel occlusions. JAMA Neurol 2013;70:831-36
6. Wikholm G. Mechanical intracranial embolectomy: a report of two cases. Interv Neuroradiol 1998;4:159-64

7. Wikholm G. Transarterial embolectomy in acute stroke. AJNR Am J Neuroradiol 2003;24:892-94

8. Broussalis E, Trinka E, Hitzl W, et al. Comparison of stent-retriever devices versus the Merci retriever for endovascular treatment of acute stroke. AJNR Am J Neuroradiol 2013;34:366-72

9. Broderick JP, Palesch YY, Demchuk AM, et al. Endovascular therapy after intravenous t-PA versus t-PA alone for stroke. $N$ Engl J Med 2013;368:893-903

10. Ciccone A, Valvassori L, Nichelatti M, et al. Endovascular treatment for acute ischemic stroke. N Engl J Med 2013;368:904-13

11. Nogueira RG, Gupta R, Dávalos A. IMS-III and SYNTHESIS expansion trials of endovascular therapy in acute ischemic stroke: how can we improve? Stroke 2013;44:3272-74

12. Pierot L, Gralla J, Cognard C, et al. Mechanical thrombectomy after IMS III, Synthesis, and MR-RESCUE. AJNR Am J Neuroradiol 2013;34:1671-73

13. Saver JL, Jahan R, Levy EI, et al. Solitaire flow restoration device versus the Merci retriever in patients with acute ischaemic stroke (SWIFT): a randomised, parallel-group, non-inferiority trial. Lancet 2012;380:1241-49

14. Kwak JH, Zhao L, Kim JK, et al. The outcome and efficacy of recanalization in patients with acute internal carotid artery occlusion. AJNR Am J Neuroradiol 2014;35:747-53

15. Zhu G, Michel P, Aghaebrahim A, et al. Computed tomography workup of patients suspected of acute ischemic stroke: perfusion computed tomography adds value compared with clinical evaluation, noncontrast computed tomography, and computed tomography angiogram in terms of predicting outcome. Stroke 2013;44: 1049-55 